\title{
AnNette Sabban \\ Zur Rolle der Phraseme für die Konstitution und Funktion des Textes. Ein Beitrag zum Konzept der textbildenden Potenzen
}

\begin{abstract}
Der Beitrag greift das Konzept der textbildenden Potenzen auf und entwickelt es weiter. Gefragt wird, inwiefern Phraseme und ihre Verwendungsweisen konstitutiv für bestimmte Dimensionen und Funktionen von Texten bzw. Teiltexten sind. Erörtert werden insbesondere: die Distribution von Phrasemtypen und Verwendungsweisen von Phrasemen in der Anzeigenwerbung im Bezug zur medialen Dimension der Texte und zu bestimmten Textfunktionen; der Einsatz von Phrasemen in überregionalen Tageszeitungen und ein darüber vermitteltes Produzentenimage; Bezüge zwischen Phrasemgebrauch und affektiven Textdimensionen, etwa in Form von pointierenden Textschlüssen, witzigen Textteilen in der Karikatur oder sich unterhaltsam gebenden Moderationen im Radio. Des Weiteren wird ein Zusammenhang angedeutet zwischen der gehäuften Verwendung von Phrasemen und distanzierend-spöttischen, ironisierenden Redeweisen in Sketch und Glosse. Die Ergebnisse geben u. a. Anlass, das prototypische Verständnis vom Text als einem ausschließlich zweckrational angelegten und vom Produkt her beschreibbaren Ganzen zu überdenken.
\end{abstract}

\section{Zur Fragestellung: Phraseme ,in Texten“ versus ,textbildende Potenzen“" von Phrasemen}

Phraseologische Untersuchungen der letzten fünfzehn Jahre sind von der grundsätzlichen Erkenntnis geprägt, dass Phraseme einen bedeutenden Anteil von Texten ausmachen. ${ }^{1}$ Das gilt insbesondere, wenn man sich nicht auf den klassischen Kernbereich der Phraseologie beschränkt, sondern die ganze Fülle fester, nicht notwendigerweise idiomatischer Mehrwortverbindungen berücksichtigt: „referentielle“, „strukturelle“ und „kommunikative“" Phraseme sowie weitere spezielle Klassen. ${ }^{2}$

Lange Zeit dominierten Fragen der Bestandsaufnahme, der Klassifikation und der Bestimmung eines invarianten Kerns von Phrasemen. Im Hinblick darauf interessierte man sich dafür, in welcher Erscheinungsform Phraseme in Texten begegneten: ob in der "tradierten Normalform“, als usuelle Variante

I Vgl. u. a. Sinclair (1991, S. 109 ff.). - Der Terminus Phrasem wird hier als Oberbegriff gegenüber Phraseologismus bevorzugt; zu einer Diskussion der Begrifflichkeit sei auf Donalies (1994, insbes. S. $346 \mathrm{f}$.) verwiesen.

${ }^{2}$ Vgl. die bei Burger (1998, Kap. 2) vorgeschlagene Basisklassifikation. 
oder als okkasionelle Variation bzw. Modifikation. Darüber hinaus interessierte man sich für die genaue Bedeutung von Phrasemen im Unterschied zu Einwortlexemen, für die Anbindung insbesondere modifizierter Phraseme an Text und Kontext und damit verbundene Verstehensprozesse sowie Aspekte ihrer Wirkung. Dabei wurden - mit variierender Benennung - die folgenden grundsätzlichen Verwendungsweisen von Phrasemen ,in Texten“ unterschieden:

1. Unmarkierte Verwendung: Phraseme als bloßer „Baustein “ des Textes. Phraseme können - und das gilt auch für den klassischen Kernbereich, die Idiome - im Text als ganz normaler „Baustein“ verwendet werden, ohne dass mit ihnen ein Mehrwert verbunden wäre. Dazu das folgende Beispiel:

(1) Während der Nacht wird der Geiselnehmer immer müder. Die Verhandlungen geraten in eine Sackgasse. Doch Oberst F. M. sowie die Verhandler A.G. und L.S. [...] geben nicht auf - auch nicht, als es Mittwoch [...] wieder kritisch wird. (Die Presse 7.7.1994; Korpus COSMAS I)

Als unmarkiert kann die Verwendung des Idioms in die/eine Sackgasse geraten hier auf Grund folgender Gegebenheiten bezeichnet werden: die bildliche Grundlage wird im Text nicht aufgegriffen; es gibt keinen Hinweis darauf, dass die Wahl genau dieses Phrasems durch ein inhaltliches oder situatives Element, mit dem seine bildliche Basis in eine Verweisbeziehung gebracht werden könnte, gleichsam angestoßen worden wäre; das Idiom ist im thematischen Zusammenhang politisch-wirtschaftlicher Verhandlungen ausgesprochen häufig: eine entsprechende Kookkurrenzanalyse über das Korpus COSMAS weist die Verbindung als statistisch auffällig aus. ${ }^{3}$ Unmarkierte Verwendungsweisen wie diese widerlegen die gelegentlich anzutreffende Ansicht, wonach Phraseme, und zwar gleich welcher Art, aus einem ganz besonderen Holz geschnitzt seien und wonach schon allein die Präsenz eines einzigen Exemplars dem Text eine besondere Qualität verleihe, ihn in irgendeinem Sinne anreichere.

2. Markierte Verwendung, Fall 1: Fokussieren der Zeichenbeschaffenheit. Kompositionalität und innere Zeichenbeschaffenheit eines Phrasems, beispielsweise die Bildlichkeit idiomatischer Phraseme, können durch ein bestimmtes Textarrangement jederzeit aufgerufen werden. In einem solchen Fall liegt eine erste markierte Verwendungsweise vor. Den Unterschied zur bloßen Baustein-Funktion zeigt der folgende Textausschnitt, in dem dasselbe Phrasem wie in (1) verwendet wird:

(2) In dem RAF-Brief heißt es unter anderem: „Es ist notwendig zu sehen, dass wir uns in einer Sackgasse befinden, um Wege aus ihr herauszufinden." (Tiroler Tageszeitung, 21.4.1998; Korpus COSMAS I)

Das Bild der Sackgasse wird mit der - übertragen zu interpretierenden - Formulierung Wege ... herausfinden im gleichen Satz aufgegriffen. Die Rekur-

${ }^{3}$ Zur genauen Bedeutung einer ,statistischen Auffälligkeit" im Rahmen der Kookkurrenzanalyse siehe Steyer (2003). 
renz der Bildebene auf engstem Raum ist ein Hinweis darauf, dass sich der Textverfasser die Beschaffenheit des Phrasems bewusst gemacht und seine Formulierung darauf abgestimmt hat; auch dem Rezipienten kann die Bildebene auf Grund dessen präsent werden.

Häufig ist auch ein Fall wie der folgende:

(3) Fußball-News direkt aufs Handy! [...] mit den brandaktuellen Vodafone-Fußball-Infos [...] bleiben Sie im ganzen Vodafone-Netz immer am Ball und wissen, wer wann wie gespielt hat. (Flyer der Firma Vodafone, Februar 2003)

Hier besteht eine Beziehung zwischen dem Idiom am Ball bleiben und einem anderen, wörtlich zu interpretierenden Ausdruck des Textes, der als Komponente im Phrasem enthalten ist bzw. mit ihm in einer formal-inhaltlichen Beziehung steht (hier: FußBall - Ball) ${ }^{4}$

Mit solchen Textarrangements - einer Art Isotopie in einem erweiterten, auf Komponenten von Mehrwortverbindungen in ihrer wörtlichen Lesart bezogenen Sinne - wird das Phrasem in seiner Beschaffenheit fokussiert. Genau damit können bestimmte Wirkungsabsichten verbunden werden.

Das Phrasem selbst bleibt äußerlich unverändert; für die Textbedeutung im engeren, referenzsemantischen Sinne ist letztlich nur die Phrasembedeutung relevant. Diese wird im Übrigen oftmals zusätzlich im Text konkretisiert und auf die jeweilige Situation bezogen (in (3): ,,bleiben Sie am Ball ... und wissen, wer wann wie gespielt hat").

3. Markierte Verwendung, Fall 2: Nutzung des Phrasems als Wortkette. Eine andersartige, ebenfalls auffällige Verwendungsweise liegt vor, wenn das Phrasem eigentlich nur als Wortkette genutzt wird. In diesem Fall ist die Phrasembedeutung für die Textbedeutung im engeren Sinne nicht relevant. Vielmehr wird eine - wörtlich zu verstehende - Formulierung genau deshalb gewählt, weil sie mit dem Wortlaut eines Phrasems zusammenfällt; dabei werden Umdeutungen der Wörtlichkeit gegenüber dem Wortlaut des Phrasems und eine unübliche Ausdrucksweise in Kauf genommen oder bewusst einkalkuliert. Beispiele:

(4) Junge Türken kommen auf den Hund

(Hildesheimer Allgemeine Zeitung, Lokalteil, 3.1.03, S. 13)

Textinhalt: Türkische Jugendliche suchen und finden einen verloren gegangenen Hund.

(5) Wir haben sie nicht alle.

(Werbeserie der taz im Jahr 2002 im Hinblick auf eine bestimmte Zahl von Abonnenten; lies: ,Wir haben [noch] nicht alle Abonnenten, die wir haben wollen.')

${ }^{4}$ Weitere Elemente, die gerne mit einer Komponente des Idioms in eine Verweisbeziehung gebracht werden, sind: ein Aspekt der Situation, in der sich der Text ereignet, sowie ein mit diesem situativen Element oder auch einzelnen Textinhalten verknüpfbares Wissenselement. 
Die Wörter werden hier nicht mehr als Komponenten einer Zeicheneinheit höherer Ordnung benutzt. Damit wird die komplexe Zeicheneinheit aufgelöst. Allerdings ist davon auszugehen, dass die Phrasembedeutung in aller Regel beim Leser aufgerufen werden soll, und auch damit können bestimmte Wirkungsabsichten verbunden sein.

Die Fälle 2. und 3., bei denen die Veränderung an der Wortfolge selbst nicht in Erscheinung tritt und sich nur ,nach innen“, auf die Bedeutung richtet, werden auch als Spielarten ,semantischer Modifikation“ bezeichnet (z. B. bei Burger 1998, S. $150 \mathrm{ff}$.).

4. Markierte Verwendung, Fall 3: Formale Modifikation. Im Unterschied zu den geschilderten Fällen 2. und 3. gibt es Modifikationen, bei denen das Phrasem in seiner äußeren Gestalt verändert wird. Diese Modifikationen sind nur dann im vollen Umfang verständlich und im Sinne der damit verbundenen Intention wirksam, wenn das abgewandelte Original durch die Variation hindurch erkannt wird. Die veränderte Beschaffenheit wird zusammen mit dem evozierten Original in vielfältiger Weise genutzt. Dazu nur stellvertretend ein einziges Beispiel:

(6) Hier in Jakutsk erblickte der Bürgermeister den Nebel der Welt. Auch sein Vater wurde hier geboren. (FAZ 3.2.1986, S. 3)

Das Gemeinsame aller auffälligen Verwendungsweisen 2., 3. und 4. liegt in Folgendem: das Phrasem wird nicht nur als Ausdruckseinheit und als Träger einer Bedeutung genutzt, sondern der Textproduzent macht sich seine Beschaffenheit zu Nutze. Das sind: (a) seine Mehrgliedrigkeit, (b) Eigenschaften des jeweiligen Phrasemtyps wie z. B. seine Idiomatizität und (c) speziellere Merkmale von Untergruppen, etwa die Bildhaftigkeit im engeren Sinne und die daraus resultierende Visualisierbarkeit der wörtlichen Ebene. ${ }^{5}$

Diese grundsätzlichen Verwendungsweisen nun sind hinlänglich beschrieben. ${ }^{6}$ Nur ansatzweise wurde dagegen versucht, einer grundlegenden textlinguistischen Frage nachzugehen: der nach einem Zusammenhang zwischen Phrasemen und ihrer Verwendungsweise einerseits und dem Text in seiner spezifischen Beschaffenheit, seiner „Konstitution“, sowie den damit verbundenen Funktionen andererseits. Als Programm wurde die Frage im Zusammenhang mit dem Konzept der "textbildenden Potenzen“" gestellt, das auf die sowjetische Phraseologie der 70er Jahre zurückgeht und von Dobrovol'skij weiterentwickelt wurde. ${ }^{7}$ Es fragt in dem eben skizzierten Sinne genau nach

\footnotetext{
5 Zur Abgrenzung von „bildhaft" und ,bildlich“" siehe Burger (1989). Die Visualisierbarkeit des Bildes spielt etwa bei der Auswahl der Bilder in Fernsehnachrichten (dazu Burger 1999) oder auch bei der Untertitelung von Bildern in den Printmedien eine Rolle.

6 Siehe umfassend Wotjak (1992), Hemmi (1994), Sabban (1998a), Balsliemke (2001); zusammenfassend Burger (1998, S. $150 \mathrm{ff}$.).

7 Grundlegend Dobrovol'skij (1980, S. 691 f.; 1987, S. 69 f.), anknüpfend an Arbeiten von Černyševa und Zaičenko aus den 70er Jahren; ferner Fleischer ( ${ }^{2} 1997$, S. 213 ff.).
} 
der Beziehung zwischen bestimmten Verwendungsweisen, „Textgestalt“ und „kommunikativer Funktion“. „Textbildend“ ist also zu verstehen als „den Text (in seiner Gestalt) ausmachend", es ist ein am Produkt, nicht am Prozess des Textherstellens orientiertes Konzept. Ein besonderer Wert wurde darauf gelegt, dass die Verwendungsweisen ihrerseits als "Potenzen“ in den allgemeinen und spezifischen sprachlichen Eigenschaften der Phraseme angelegt sind.

Allerdings ist dieses Konzept insofern Programm geblieben, als der Schwerpunkt auf eine Beschreibung der Verfahren gelegt und die Gedanken nur an einzelnen Beispielen erläutert wurden. Außerdem wurden unmarkierte Verwendungen von Phrasemen als ,normale Bausteine“ des Textes ausgeklammert, da sie als "unterste Stufe" textbildender Potenzen offenbar nicht weiter interessant waren. Wenn Phraseme jedoch auch normale Bausteine des Textes sind, dann ist es sinnvoll zu fragen, ob es Zusammenhänge zwischen der Art der Bausteine, also Typen von Phrasemen, und dem Text und den damit verbundenen Funktionen gibt.

Im Folgenden soll - unter Beschränkung auf schriftliche Texte - gezeigt werden, wie man sich der Frage systematischer nähern kann. Dabei werden auch Ergebnisse bisheriger Arbeiten aus dieser Perspektive betrachtet. Das Anliegen des Beitrags lässt sich in folgende These fassen:

Phraseme (als Exponenten bestimmter Phrasemtypen) und bestimmte Verwendungsweisen von Phrasemen können für Texte konstitutiv in folgendem Sinne sein: sie leisten einen entscheidenden Beitrag zu Texten oder Teiltexten in ihrer jeweiligen Beschaffenheit und den damit realisierten Funktionen; im besonderen Fall konstituieren sie selbst den Text als solchen. ${ }^{8}$

\section{Korrelationen zwischen Phrasem und Text: quantitative Untersuchungen}

\subsection{Phrasemtyp und Textsorte}

Eine erste Frage gilt möglichen Korrelationen zwischen Phrasemtyp und Textsorte. Diese Frage wurde bei Dobrovol'skij gestellt, die Möglichkeit zur „Aufstellung einer strengen Korrelation“ allerdings bezweifelt (1987, S. 72 ff.). Um eine strenge Korrelation kann und soll es auch gar nicht gehen; ebensowenig wird davon ausgegangen, dass eine solche Frage für jeden

${ }^{8}$ Außer Acht bleiben spezielle Klassen von Phrasemen, die sich gerade durch die Realisierung bestimmter Aufgaben ,für den Text" definieren. Es sind dies die deutlich auf den Prozess des Textherstellens bezogenen Gruppen der,,metadiskursiven“ und „textorganisierenden" Phraseme. Metadiskursive Wendungen (im doppelten Sinne; nennen wir es darum ruhig ..., ... ist das richtige Wort u. a. m.) explizieren einen Aspekt des Textherstellens; ihr Einsatz beruht auf der „Fähigkeit des Sprechers, beim Reden einen bewertenden Rückblick auf das Diskursmaterial zu werfen" (Cortès 2001, S. 44). Textorganisierende mehrgliedrige Ausdrücke in schriftlichen Texten (,macro-organizers") werden ansatzweise z. B. bei Granger (1998, S. $154 \mathrm{ff}$.) untersucht. 
Phrasemtyp sinnvoll wäre. Für einige Phrasemtypen jedoch und für bestimmte, relativ fein differenzierte Textsorten ist eine Korrelation tendenzweise möglich.

Ermittelt werden können derartige Zusammenhänge durch ein quantifizierendes Vorgehen. Entsprechende Ergebnisse finden sich bei Hemmi (1994), die Werbungen in verschiedenen Medien vergleichend untersucht und - unter anderem - die medienspezifische Distribution von Phrasemtypen ermittelt hat. Besonders deutliche Tendenzen sind bei zwei Phrasemtypen bzw. Untertypen erkennbar: ${ }^{9}$

\begin{tabular}{|l|l|l|}
\hline $\begin{array}{l}\text { Textsorte } \\
\text { Vergleichsdimension: } \\
\text { Medium }\end{array}$ & $\begin{array}{l}\text { Phrasemtyp 1: } \\
\text { Routineformeln }\end{array}$ & $\begin{array}{l}\text { Phrasemtyp 2: Geflügelte } \\
\text { Worte - unterteilt nach } \\
\text { Quellbereich }\end{array}$ \\
\hline Anzeigenwerbung & selten & vorwiegend aus Literatur \\
\hline Radiowerbung & häufig & vorwiegend aus Musik \\
\hline TV-Werbung &..$^{10}$ & vorwiegend aus Film \\
\hline
\end{tabular}

Tabelle 1

Routineformeln sind in der (schriftlichen) Anzeigenwerbung ausgesprochen selten. Umso häufiger sind sie in Radiowerbungen: hier sind sie ein typischer „Baustein“ für die darin inszenierten Gesprächs- und Interviewsituationen; darüber hinaus gehören sie zu den radiospezifischen Möglichkeiten der Aufmerksamkeitssteuerung: manche von ihnen dienen der direkten Ansprache des Hörers.

Beim Typ Geflügelte Worte erweist es sich als aufschlussreich, die Ausdrücke nach Herkunftsbereichen weiter zu untergliedern. Dann zeigt sich, dass Geflügelte Worte bevorzugt aus den Quellen stammen, die dem jeweiligen Medium am nächsten stehen (Hemmi 1994, S. 87). So werden im Printmedium Zeitschrift besonders gerne Werke der Literatur zitiert, in der Radiowerbung Musiktitel, in der Fernsehwerbung Filmtitel.

Es lässt sich also festhalten, dass bestimmte Typen von Phrasemen in medial unterscheidbaren Textsorten unterschiedlich häufig sind; zumindest gibt es partiell und in derTendenzunterschiedliche Präferenzen. Bezogen auf die Ausgangsthese bedeutet dies: die Distribution der Phraseme ist in einem gewissen Ausmaß den Medieneigenschaften angepasst; insofern tragen Phraseme zur Konstitution des Textes, zu seiner medienspezifischen Beschaffenheit, bei.

Dieses Ergebnis ist insofern von allgemeinerer Bedeutung, als es zeigt, dass die „situative Einbettung" - das ist in diesem Fall die mediale Umgebung, in der sich der Text ereignet - dem Text nicht äußerlich bleibt, sondern

${ }^{9} \mathrm{Zu}$ den Details der Distribution siehe Hemmi (1994, S. $64 \mathrm{ff}$.).

${ }^{10}$ Das Zeichen ./. bedeutet, dass keine eindeutige Tendenz erkennbar ist oder dass eine genaue Angabe für den vorliegenden Argumentationszusammenhang verzichtbar ist. 
in die Wahl bestimmter Ausdrucksmittel hineinwirkt, und das vermittelt über die jeweiligen Verfasser des Textes, ihre Ausbildung, ihr Wissen oder auch ihre Annahmen über die Interessen der Adressaten.

\subsection{Verwendungsweisen von Phrasemen in ihrer Beziehung zu Teil- texten - am Beispiel der Anzeigenwerbung}

Eine zweite Frage gilt möglichen Zusammenhängen zwischen bestimmten Verwendungsweisen von Phrasemen und Texten. Die Frage richtet sich also auf den Beitrag bestimmter Verwendungsweisen zur charakteristischen Beschaffenheit eines Textes, und das im Hinblick auf die Realisierung der mit dem Text verbundenen Funktionen.

Auch für die Untersuchung dieser Frage eignet sich eine Zählung. Damit diese praktikabel ist, können die in Abschnitt 1 unterschiedenen Verwendungsformen - welche ihrerseits bereits eine Abstraktion der tatsächlichen Vielfalt darstellen - allerdings nur in zwei relativ grobe Klassen unterteilt werden: nicht-modifizierte, unmarkierte Phraseme und modifizierte Phraseme.

Modifizierte Phraseme sind in der Anzeigenwerbung besonders häufig; das ist ein Gemeinplatz in der Phraseologieforschung; Auszählungen von Hemmi (1994) und Balsliemke (2001) führen dies noch einmal deutlich vor Augen. ${ }^{1}$ Aufschlussreich ist es nun, wie im Übrigen auch für andere textlinguistische Fragestellungen, wenn man es nicht bei einer Betrachtung des

\begin{tabular}{|l|l|l|}
\hline $\begin{array}{l}\text { Anzeigenwerbung: } \\
\text { Makrostruktur'2 }\end{array}$ & $\begin{array}{l}\text { Erscheinungsform des } \\
\text { Phrasems (Tendenzen) }\end{array}$ & $\begin{array}{l}\text { übergeordnete Funktion } \\
\text { der Teiltexte }\end{array}$ \\
\hline 1. Schlagzeile & $\begin{array}{l}\text { sehr oft modifiziert } \\
\text { (2mal soviel wie im Slogan) }\end{array}$ & $\begin{array}{l}\text { appellierend, Aufmerksam- } \\
\text { keit weckend }\end{array}$ \\
\hline 2. Fließtext/Haupttext & nicht modifiziert & $\begin{array}{l}\text { oft erklärend-informativ, } \\
\text { enthält oft Produktbe- } \\
\text { schreibung }\end{array}$ \\
\hline 3. Slogan & gelegentlich modifiziert & $\begin{array}{l}\text { appellierend; mnemotech- } \\
\text { nisch }\end{array}$ \\
\hline
\end{tabular}

Tabelle 2

${ }^{11}$ Eine sämtliche Typen von Phrasemen umfassende Zählung von Hemmi (1994, S. 102) zeigt: etwa die Hälfte der Phraseme in der Anzeigenwerbung erscheint in der Normalform oder einer usuellen Variante, die andere Hälfte in modifizierter Form. Nach Balsliemke (2001, S. 185) liegt der Anteil modifizierter Phraseme in der Textsorte Anzeigenwerbung noch höher: es finden sich etwa eineinhalbmal so viele modifizierte wie nicht-modifizierte Phraseme, wenn man nur diejenigen berücksichtigt, die normalerweise auch tatsächlich abgewandelt werden können, wenn man also adverbiale Phraseme wie frei Haus/in Ruhe aus der Zählung ausklammert. Hier ergibt sich ein Rückbezug zur Beschaffenheit und daraus resultierenden Eignung verschiedener Phraseme (den „Potenzen“) für eine modifizierte Verwendung. - Vergleiche mit anderen Textsorten, die nach demselben Verfahren ausgezählt worden wären, liegen nicht vor.

12 Zur Makrostruktur gehört auch das Bild bzw. „Visual“, das erstmals bei Balsliemke (2001) systematisch in die Untersuchung miteinbezogen wird. 
Gesamttextes belässt, sondern die verschiedenen Teile des Textes berücksichtigt. Texte der Anzeigenwerbung eignen sich dafür in besonderer Weise, da sie in der Regel eine klare Makrostruktur aufweisen. Zählungen bei Balsliemke (2001, S. $103 \mathrm{ff}$.) ergeben, dass sich die Verwendungsweisen unterschiedlich auf die Teile des Anzeigentextes verteilen. Diese unterschiedlichen Tendenzen der Distribution legen einen Zusammenhang mit den jeweiligen Funktionen nahe.

Im Fließtext finden sich vor allem nicht-modifizierte Phraseme (Balsliemke 2001, S. $106 \mathrm{f}$.). Dieser Teiltext enthält in der Regel Produktinformationen; seine Funktion ist typischerweise informierend-erklärend. ${ }^{13}$ Schlagzeile und Slogan dagegen, vergleichsweise exponierte Teile des Textes, sind charakteristische Orte für die Platzierung modifizierter Phraseme; dabei enthält die Schlagzeile doppelt so viele modifizierte Phraseme wie der Slogan (Hemmi 1994, S. 176f.); die Häufigkeit im Slogan ist nach Balsliemke (2001, S. 105) sogar insgesamt als eher gering zu bewerten. Eine wesentliche Leistung modifizierter Phraseme in der Schlagzeile besteht darin, die Aufmerksamkeit des Betrachters zu gewinnen - Grundvoraussetzung für die Wahrnehmung des Werbetextes überhaupt und daher ein wesentliches Teilziel der Werbung, welches seit langem in der Marktforschung benannt ist (vgl. Sowinski 1998, S. 30). Die "textbildende Potenz" mancher Phraseme liegt somit genau darin, dass sie auf Grund ihrer Beschaffenheit für Verwendungsweisen geeignet sind, diese spezifische Funktion eines Teiltextes zu realisieren.

\section{Gleichsinnige Ergebnisse in nicht-quantitativen Untersuchungen}

Die in quantitativen Untersuchungen ermittelte Distribution modifizierter Phraseme und insbesondere die Deutung der damit verbundenen Funktionen wird durch andere, nicht-quantitative Untersuchungen bestätigt.

So ist wiederholt beobachtet worden, dass sich im Berichtsteil der Zeitung vergleichsweise wenige modifizierte Phraseme finden. Einen funktionalen Zusammenhang formuliert schon Cowie (1991, S. 103) für das Englische, wenn er sagt, dass sich Phraseme in Normalform, auch Kollokationen und usuelle Metaphern, für die „schmucklose Präsentation von Fakten“"14 eignen, also für eine informierend-erklärende Funktion. In Zeitungskommentaren und Leitartikeln dagegen sind modifizierte Phraseme vergleichsweise häufig; dies ist der Ort, an dem Ereignisse perspektiviert dargestellt und bewer-

13 Warum Phraseme im Fließtext relativ häufig auftreten (zu Zahlen Balsliemke 2001, S. 105; ähnlich Hemmi 1994, S. 107), welche Funktionen sie dort genau haben, lässt sich auf Grund einer Zählung allein nicht näher bestimmen, da die Phraseme zu heterogen sind. Wenn Hemmi (1994) derartige Passagen als sachlich, aber dennoch ,aufgelockert“ beschreibt, so hat sie dabei vermutlich vor allem idiomatische Ausdrücke im Blick.

14 Wörtlich: „the unadorned presentation of facts", Cowie (1991, S. 103). 
tet werden (ebda., S. 103; auch Blumenthal 1999). Phraseme eignen sich auf Grund ihrer spezifischen Bedeutungsnuancen, ihres oft konnotativ-wertenden Mehrwerts und ihrer Expressivität insgesamt gesehen dazu, diese kommentierende Funktion wahrzunehmen oder zumindest zu ihrer Realisierung beizutragen.

Untersuchungen zur Plakat- und Anzeigenwerbung zeigen, dass modifizierte Phraseme nicht nur in der Schlagzeile vorkommen, sondern dass sie oftmals als Schlagzeile verwendet werden, dass sie die Schlagzeile praktisch konstituieren. Darüber hinaus lassen sich hier Strategien ausmachen, für die sich bestimmte Untergruppen von Phrasemen dank ihrer semantischen und pragmatischen Merkmale in besonderer Weise eignen. Eine auffällig häufig eingesetzte Strategie ist die der sogenannten ,inszenierten Negativität" (Sabban 1998b, 1998c). Dabei werden semantisch negativ gerichtete, komplexe Ausdrücke und Ausdrucksmuster verwendet, d.h. Ausdrucksweisen, die üblicherweise recht rüde Sprechakte vollziehen (siehe (7), (8)), oder es werden, ganz gegen die üblichen pragmatischen Spielregeln, negative Aussagen über die eigene Person getroffen (siehe (9)):

(7) Sie können uns im Mondschein begegnen.

(Nachtliniennetz der Münchner Verkehrsbetriebe, Dezember 1994)

(8) Stecken Sie sich ihr Sparbuch doch dorthin, wo es hingehört!

(Werbung einer Bank, Hildesheim, Februar 1996)

(9) Wir haben sie nicht alle!

(Werbeserie der $t a z, 2002$; siehe auch oben, (5))

Die Werbestrategie beruht auf einem ,pragmatischen Paradox“: der Werbende will den Kunden umwerben, beleidigt ihn jedoch; der Produzent will für sich werben, tut aber wiederum etwas dafür gänzlich Ungeeignetes, indem er etwas Vernichtendes über sich selbst sagt. Jedenfalls dürfte dies die jeweils erste Interpretation sein, die sich dem Betrachter spontan aufdrängt. (Zwar entzieht sich dies der Beobachtung, es kann aber auf Grund psycholinguistischer Untersuchungen zum Verstehen von Phrasemen als wahrscheinlich angenommen werden.) Die negativ gerichtete Phrasembedeutung erweist sich freilich - spätestens im Verlauf der Lektüre - als letztendlich nicht gemeint, der Ausdruck soll vielmehr in irgendeiner Weise wörtlich genommen werden. Dazu muss der Ausdruck oftmals gegen den Strich eingefahrener Gewohnheiten gelesen, übliche Kollokationen oder Grammatisches müssen außer Kraft gesetzt werden. Der damit vermutlich verbundene kognitive Aufwand erhöht die Verweildauer des Lesers beim Text; das dient ebenfalls den Zwecken der Werbung, da es die Erinnerung an das Gelesene befördert. Die letztlich gemeinte Lesart ist die wörtliche, und diese enthält wiederum stets eine positive Aussage über das Produkt. So wird beispielsweise in (7) für den Nachtlinienverkehr der Busse und U-Bahnen geworben: man kann ihnen ,im Mondschein begegnen“. 
Bei dieser Strategie handelt es sich um eine Spielart semantischer Modifikation, einer ambiguierenden Verwendungsweise (siehe S. 240, 3.); ihre Wirkung beruht auf dem bloßen Evozieren einer Bedeutung, die für die Textbedeutung im engeren Sinne nicht relevant ist. ${ }^{15}$ Diese Nutzung sprachlicher Mittel ist mit einem affektiven Potential verbunden: der Rezipient ist verwundert, vielleicht zunächst tatsächlich negativ berührt, beim Umschlagen der Interpretation möglicherweise erheitert. Der spielerisch-provokative Gestus gegenüber dem Leser, der saloppe Umgangston und die gekonnte Verwendung von Sprache - die sich mit einem Phrasem ökonomisch vorführen bzw. realisieren lässt - stehen auch im Dienste der Image-Werbung. Sie sind geeignet, den Leser für die Firma oder das Produkt zu vereinnahmen. Intentionen der Werbung und Einsatz sprachlicher Mittel - insbesondere von Phrasemen - gehen hier eine enge Verbindung ein, genau im Sinne der ,textbildenden Potenzen“.

\section{Phrasemgebrauch und Produzentenimage - am Beispiel überregionaler Tageszeitungen}

Das eben beschriebene Etablieren eines Image über einen bestimmten Sprachgebrauch, bei dem ein spezifischer Umgang mit Phrasemen eine wesentliche Rolle spielt, ist nicht auf die Werbung beschränkt: Ähnliches ist auch bei Zeitungen zu beobachten. Wie kann dies untersucht werden? Geeignet ist ein Vergleich von Texten unter weitgehender Isolierung der interessierenden Variablen. Ein Beispiel für ein solches Verfahren, wenngleich mit anderer Zielsetzung, liegt der Untersuchung von Cowie (1991) zu Grunde; hier wurden Texte verschiedener Textsorten, Zeitungsberichte und Zeitungskommentare, zu ein und demselben Thema verglichen. Ziel war es, eine eventuell unterschiedliche Verteilung von Phrasemtypen zu ermitteln. Dabei wurde potentiellen Unterschieden zwischen verschiedenen Tageszeitungen allerdings nicht nachgegangen; die Textsorten wurden also gleichsam unabhängig vom jeweiligen Textproduzenten betrachtet. Genau diese Variable lässt sich jedoch, im Sinne einer textpragmatischen Orientierung, berücksichtigen, indem man die sprachlichen Mittel, insbesondere den Einsatz von Phrasemen, in der Berichterstattung zum gleichen Ereignis oder Thema in verschiedenen Zeitungen untersucht.

Einen ersten Ansatzpunkt bietet der Vergleich von Überschriften. Eine explorative Studie überregionaler Zeitungen, die über den Zeitraum einer Woche durchgeführt wurde, ergab Parallelbelege des folgenden Typs: ${ }^{16}$

$15 \mathrm{Vgl}$. auch das Konzept der ambiguité allusive bei Landheer (1989) und dessen Dynamisierung durch Einbeziehung des zeitlichen Verlaufs der Textrezeption bei Sabban (2001).

${ }^{16}$ Die zitierten Belege stammen alle vom 16. oder 17.1.2003; auf eine genaue Angabe wird aus Gründen der Übersichtlichkeit verzichtet. Die explorative Untersuchung würde an Hand einer größeren Datenmenge überprüft werden müssen. 


\begin{tabular}{|c|c|}
\hline Die Tageszeitung (taz) & Andere überregionale Zeitungen \\
\hline (10) & \\
\hline $\begin{array}{l}\text { Rosarote Zeiten bei Opel } \\
\text { (11) }\end{array}$ & $\begin{array}{l}\text { - Opel kann seinen Verlust verringern (DIE WELT) } \\
\text { - Opel will bald Gewinn machen (Süddeutsche Zeitung) }\end{array}$ \\
\hline $\begin{array}{l}\text { Lufthansa hebt nicht } a b \\
\text { (12) }\end{array}$ & - Warnstreiks bei Lufthansa (Süddeutsche Zeitung) \\
\hline Warnstreiks machten & - Lufthansa streicht wegen Warnstreiks 40 Flüge (DIE WELT) \\
\hline 40 flieger [sic] flügellahm & $\begin{array}{l}\text { - Warnstreiks treffen Lufthansa (Süddeutsche Zeitung) } \\
\text { - „Wir hoffen, dass der Lufthansa-Vorstand unser Signal } \\
\text { versteht“ (Frankfurter Rundschau) }\end{array}$ \\
\hline & \\
\hline $\begin{array}{l}\text { Ein EU-Chef mehr } \\
\text { oder weniger } \\
\text { (siehe auch den 1. Satz } \\
\text { des Textes: Ein Doppel- } \\
\text { kopf soll es werden.) }\end{array}$ & $\begin{array}{l}\text { - EU-Doppelspitze stößt auf Skepsis (Frankfurter Rundschau) } \\
\text { - Paris und Berlin für eine europäische „Doppelspitze“ (FAZ) } \\
\text { - Doppelspitze soll künftig die EU führen (Süddeutsche Zei- } \\
\text { tung) } \\
\text { - Berlin und Paris einig über EU-Doppelspitze (DIE WELT) }\end{array}$ \\
\hline
\end{tabular}

Tabelle 3

Diese Auswahl weist auf Unterschiede zwischen der taz und anderen überregionalen Zeitungen hin. Die Überschriften in der taz arbeiten häufig mit „auffälligen Verwendungsweisen“ im beschriebenen Sinne. So macht sich die Überschrift (10) die Farbenmetaphorik dreier Phraseme bzw. ihrer Komponenten zu Nutze: der roten Zahlen kaufmännischer Bilanzen, der rosaroten Brille, durch die man etwas sieht, und des rosaroten Lichts, in dem man etwas erscheinen lässt (Duden Band 11). Daraus schafft die taz auf gekonntprofessionelle Weise eine eigene Formulierung. Die Konnotationen der mitverwendeten und beim Leser potentiell evozierten Ausdrücke (etwas in rosarotem Licht erscheinen lassen) sind eine wesentliche Komponente des distanzierend-ironischen Darstellungsmodus, mit dem die taz politische Ereignisse und gesellschaftliche Phänomene generell darstellt und kommentiert. In gleicher Weise vermittelt (13), Ein EU-Chef mehr oder weniger, in der die Wörter mehr und weniger auf Grund des Kontextes ihr volles semantisches Gewicht erhalten, dank des evozierten Phrasems mehr oder weniger eine gewisse distanzierte Lässigkeit. Diese Grundhaltung ist ein wesentlicher Teil des Image dieser Zeitung, das sie über ihre Art, Sprache zu gebrauchen, Tag für Tag aufs Neue etabliert. Die anderen überregionalen Zeitungen verfahren dagegen durchweg rein sachorientiert.

Die exemplarische Zusammenstellung in Tabelle 3 macht ein Weiteres deutlich: Es werden nicht nur Phraseme, sondern auch polyseme, ambiguierbare Einzelwörter und Komposita in prinzipiell ähnlicher Weise auffällig genutzt und teilweise gegenüber ihrer normalen Verwendung umgedeutet (siehe (11) und (12)). Phraseme sind also nur ein, wenngleich wichtiges und besonders auffälliges, Element eines bestimmten Darstellungsmodus. 


\section{Phrasemgebrauch und affektive Textdimensionen: Vergnügen am Text und Unterhaltungswert}

Bisher war von ,textbildenden Potenzen“ der Phraseme insbesondere für die Gestaltung von Werbeschlagzeilen und Zeitungsüberschriften die Rede. Typischerweise handelt es sich dabei um durch ambiguierende Verwendungsweisen oder auch um Formulierungen, die in erkennbarer Weise auf etablierten Phrasemen basieren, indem sie sich deren Wortlaut oder Metaphorik zu Nutze machen.

Solche Verwendungsweisen eignen sich nun auch für die Konstituierung anderer Textteile, Teiltexte und auch ganzer Texte. Davon soll im Folgenden die Rede sein. Der gemeinsame Nenner der ausgewählten Fälle besteht in verwandten affektiven Wirkungen, die dank des spezifischen Einsatzes von Phrasemen mit dem Text verbunden werden: einer Erheiterung des Rezipienten bei der Lektüre eines auf einem bestimmten Phrasemgebrauch gründenden Witzes oder einem Vergnügen am Formulieren auf Seiten des Textproduzenten.

\subsection{Pointierender Textschluss}

Phraseme eignen sich zur Konstituierung eines pointierenden Textschlusses, also eines bestimmten Textteils mit einer bestimmten Qualität und Wirkung. Dazu folgendes Beispiel aus der Rubrik „Was fehlt“ im Nachrichtenteil der taz, einer Rubrik, die vielfach kuriose Meldungen enthält:

(14) Geglückter Bankraub

Es war wohl nicht sein Tag. Nach dem Überfall auf die [...] Bank [...] hinterließ der Räuber [...] eine Spur aus 100-Dollar-Scheinen. Beim Fluchtwagen angekommen, sah er die Schlüssel im verschlossenen Auto liegen. [...] Also flüchtete er zu Fuß. Als Passanten ihn überwältigen wollten, zog der Mann eine Waffe. Damit schoss er sich selbst ins Bein. Dazu hat er eine Anklage wegen Raubes und versuchten Mordes am Hacken. (taz, 20.01.03, S. 2)

Der Text zählt chronologisch und sachlich registrierend eine Serie von Katastrophen auf - gewissermaßen eine Konkretisierung von Murphy's Law If anything can go wrong it will. Der letzte Satz nun steht außerhalb der eigentlichen Ereigniskette, bringt aber inhaltlich eine Steigerung, eingeleitet mit dazu (im Sinne von, obendrein'). Seinen pointierenden Charakter erhält der Satz wesentlich durch die sprachliche Form des Ausdrucks: die gezielte Kontrastierung zwischen wörtlicher, konkreter Ebene im vorausgehenden Satz (der Bankräuber schoss sich ins reale Bein) und dem übertragen zu lesenden Ausdruck etwas am Hacken haben, dessen Bild an die Wörtlichkeit des Vorsatzes anschließt (Isotopie im erweiterten Sinne, siehe oben, S. 240). Dieser Kontrast hat eine komische Wirkung. Darüber hinaus wird das Bild aktivierbar und kann den Rezipienten zu einer irgendwie gearteten Visualisierung 
des Bildes anregen: er kann sich die Szene ,ausmalen“, die Phantasie „spielen" lassen.

Die Formulierung gewinnt eine zusätzliche Dimension dadurch, dass die Wendung etwas am Hacken haben möglicherweise gar keine usuelle Verbindung, sondern eine okkasionelle Neubildung ist: sie ist weder im Duden Band 11 noch in der Deutschen Idiomatik von Schemann verzeichnet. Dennoch kann ihr relativ problemlos ein Sinn zugeordnet werden. ${ }^{17}$

Eine gleichartig konstruierte Pointe unter Verwendung des Phrasems $\mathrm{Haa}$ re auf den Zähnen haben findet sich in folgendem Textausschnitt, der als Zitat in einer anderen Zeitung wiedergeben ist. Es kann vermutet werden, dass der Ausschnitt gerade wegen der Pointe als zitierwürdig empfunden wurde:

(15) Anlässlich des Gerichtsurteils zu Äußerungen über die Farbechtheit der Haare von Kanzler Schröder werden die Stimmen anderer Zeitungen zitiert:

Jedenfalls wissen wir nun: Schröder hat farbechte Haare, er hat sie auf dem Kopf. Und er trägt sie stets sehr schön im Kameralicht. Und die auf den Zähnen, die sieht man vor Gericht. (Rhein-Neckar-Zeitung, zitiert in der taz vom 17.5.02, S. 4, Rubrik Das dröge Thema)

\subsection{Phraseme in der Karikatur}

Die in 5.2 beschriebenen Pointen enthalten Angebote zur Visualisierung durch den Leser. Bilder können aber auch für den Leser in Form einer Zeichnung visualisiert werden. In den folgenden Karikaturen wird zeichnerisch eine Situation entworfen, in deren Textanteil die Verwendung eines Phrasems durch die dargestellten Akteure einen Witz erzeugt, somit für eine typische Funktion von Karikaturen konstitutiv ist. ${ }^{18}$

Ein für die humoristische Wirkung verantwortliches Grundschema besteht darin, dass die Verwendung des Ausdrucks - oder auch nur das damit Evozierte - in einer bestimmten Situation nicht passend ist, zum Beispiel weil das Bezeichnete zur Lösung eines Problems fundamental ungeeignet ist. Die Darstellung entlarvt den dargestellten Sprecher oder Handelnden als dumm

17 Vermutlich handelt es sich um eine eigenständige Formulierung, bei der mehrere Phraseme mit demselben Bildkern und/oder ähnlichen Konstruktionen verschränkt werden, die darüber hinaus im weitesten Sinne bedeutungsähnlich sind: sich an jds Hacken hängen ,jdm hartnäckig verfolgen'; jdm [dicht] auf den Hacken sein/ sitzen ,hinter jdm her sein, jdn verfolgen'; Dreck am Stecken haben, sich etwas zu Schulden haben kommen lassen' (Duden Band 11); jdm etwas ans Bein basteln (nicht im Duden Band 11) ,jdm einen Fehler zuschreiben (den dieser gar nicht begangen hat) ${ }^{\circ}$. - Zur Frage, warum man einer okkasionellen Neubildung wie dieser einen Sinn zuordnen kann, siehe Häcki Buhofer in diesem Band.

${ }^{18}$ Das Fischer Lexikon Publizistik (1995, S. 115) nennt folgende Funktionen von Karikaturen: belustigen (in Form von Witzzeichnungen), kommentieren (durch Bildkommentare, editorial cartoons) und angreifen (Kampfbilder). 
oder einfältig. Das wiederum bietet dem Berichtenden oder dem Zeichner (dem „Textproduzenten“) die Möglichkeit, sich über diese Person lustig zu machen und den Adressaten darin miteinzubeziehen. So mit folgender Witzpostkarte:

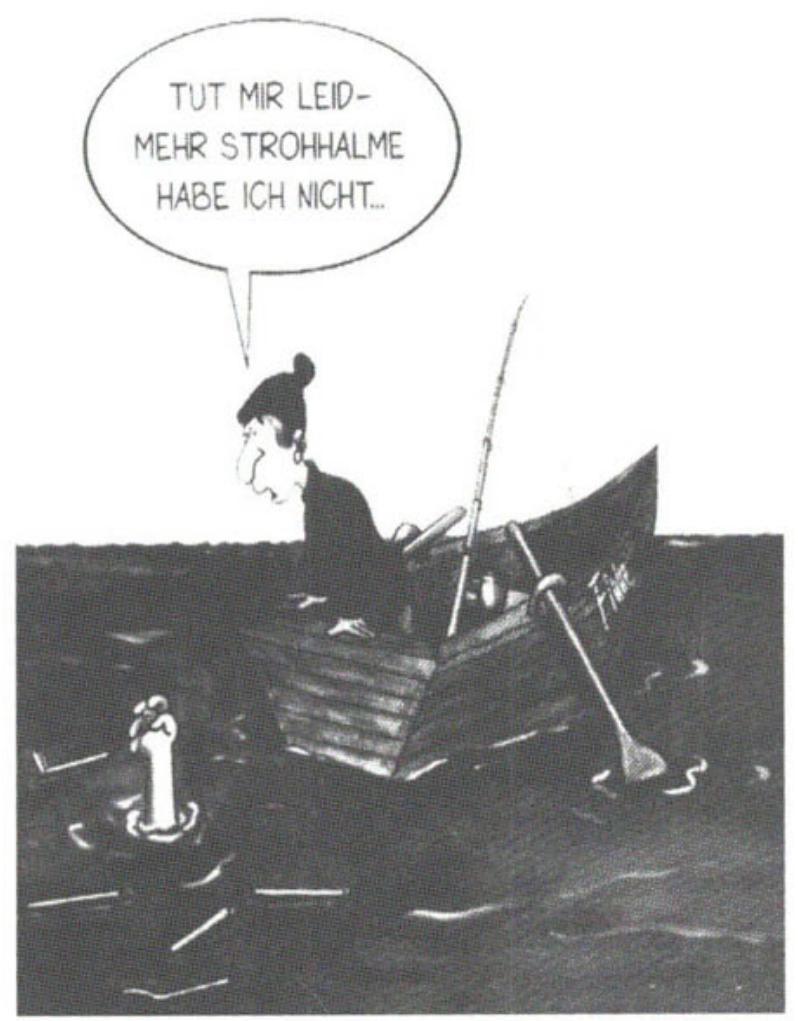

Cartoon: Harald Finke;

Postkarte Rannenberg \& friends, Hamburg

Diese Witzzeichnung beruht auf dem naiven Wörtlichnehmen eines Ausdrucks, dessen Bildkern einem der folgenden Phraseme entnommen ist: sich an jeden Strohhalm klammern; nach dem rettenden Strohhalm greifen; der rettende Strohhalm (Duden Band 11). Die Naivität resultiert daraus, dass die Person im Boot das Sprachbild nicht als solches versteht, sondern den Ausdruck wörtlich nimmt und das wörtlich Genommene auch noch in eine surreal-unsinnige Handlung überführt: den Versuch, einen Ertrinkenden mit Strohhalmen zu retten.

In der Zeitung, insbesondere im politischen Teil, ist die Karikatur eine bildliche Form des Kommentars. Der Textanteil einer Karikatur wird oft mit Phrasemen gestaltet, wie im folgenden Beispiel einer politischen Karikatur anlässlich der Entlassung Scharpings aus dem Amt des Verteidigungsminis- 


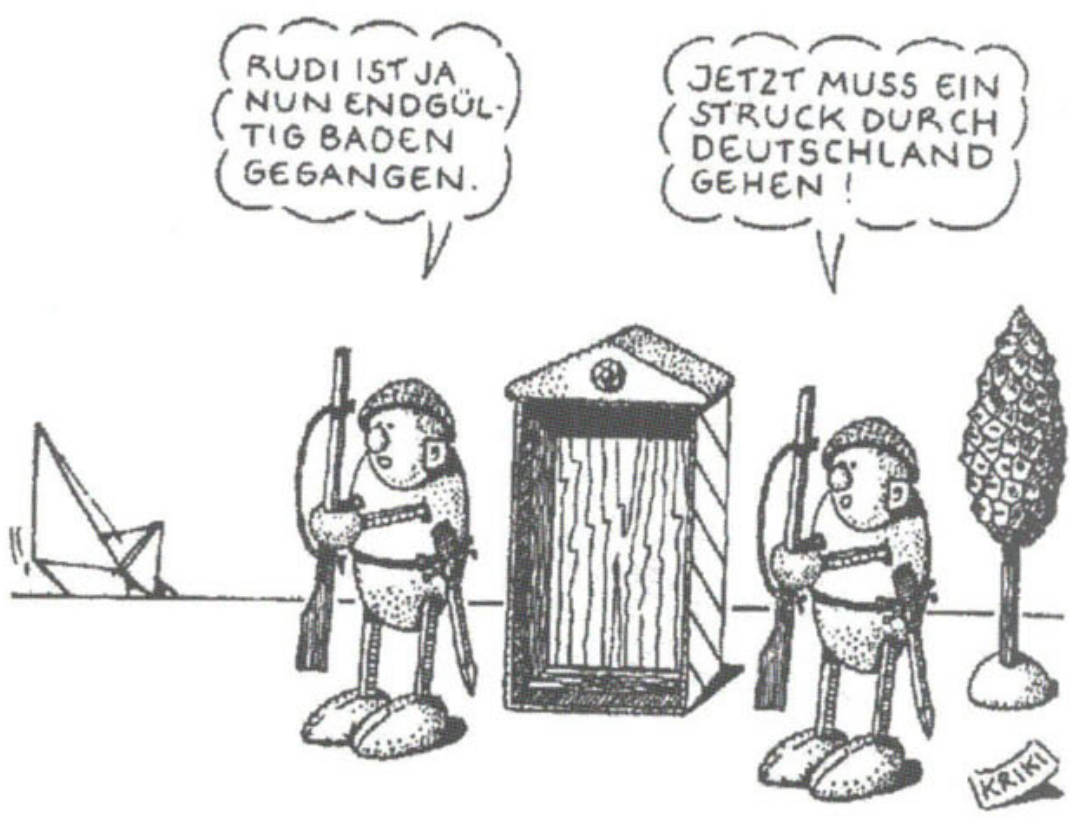

taz. 20./21.7.02, S. 11

ters und der Ernennung Strucks zu seinem Nachfolger. Das Bild zeigt zwei Wache schiebende Soldaten. Der Dialog zwischen ihnen wird konstituiert durch zwei Phraseme, die beide auffällig, modifiziert verwendet sind. Damit wird auf zwei vergangene, tagespolitisch relevante Ereignisse angespielt - diesbezüglich im Übrigen passend zur innenpolitischen Rubrik der Zeitung, in der die Karikatur steht. Das Phrasem in der Rede des linken Soldaten, (mit etwas) baden gehen, ist in Bezug auf das tagespolitische Ereignis, die Entlassung Scharpings aus dem Amt, ungefähr in seiner idiomatischen Bedeutung zu interpretieren (es ist leicht versetzt gegenüber seinem üblichen Gebrauch); auf der wörtlichen Ebene spielt es auf den Badeurlaub Scharpings auf Mallorca an. Die Rede des rechten Soldaten besteht aus einer okkasionellen Modifikation eines auf Roman Herzog zurückgehenden Ausspruchs aus dem Jahre 1998, Es muss ein Ruck durch Deutschland gehen. Verstanden werden die Anspielungen nur vom tagespolitisch informierten Leser. Dieser kann sich, und hier liegt wiederum ein affektives Moment, über den Anspielungsreichtum der Äußerungen amüsieren, unabhängig davon, ob er diese nun als geistreiche Bemerkungen der Soldaten einstuft oder als in der Situation „unfreiwillig komisch“. Unfreiwillig komisch sind Äußerungen dann, wenn sie unabsichtlich mehr implizieren als vom Sprecher gewollt; der Sprecher weiß sozusagen nicht, was er eigentlich sagt. Derartige Äußerungen sind ein häufiger Anlass von Belustigung im Alltag; sie stellen 
oft erzählenswerte Ereignisse dar..$^{19}$ Der Leser, der mehr weiß, kann sich informiert und dadurch aufgewertet fühlen; hierin liegt ein weiteres affektives Moment.

In diesem Dialog zeigt sich schließlich ein weiterer grundsätzlicher Aspekt der Text-Bildung durch Phraseme. Nur durch diese besondere Art der Darstellung, die entscheidend durch einen bestimmten Umgang mit Phrasemen konstituiert wird, ist die erneute Wiederaufnahme eines Inhalts überhaupt gerechtfertigt; der Inhalt selbst ist zu diesem Zeitpunkt längst bekannt. Anders gesagt: doppelt lesbare Phraseme ermöglichen einen Modus der Darstellung, der dank des Vergnügens, das er bereitet, den Text in seiner Existenz überhaupt erst rechtfertigt.

\subsection{Kohäsive Brücken als textkonstituierendes Prinzip: vom „aufgelockerten“ zum „lockeren“ Text}

Ein bekanntes Grundprinzip der Verwendung von idiomatischen Phrasemen ist das Wörtlichnehmen (siehe Abschnitt 1., Fall 3.). Das Phrasem wird nur als „Baumaterial“, als Wortkette, also ausdrucksseitig genutzt; die Phrasembedeutung wird gleichsam abgespalten, sie ist zumindest für die Textbedeutung nicht relevant. Gleichwohl kann sie evoziert und in unterschiedlicher Weise für die Wirkung des Textes relevant werden. Eine besondere Spielart wurde im Zusammenhang mit der inszenierten Negativität diskutiert (siehe Abschnitt 3., Beispiele (7) bis (9)). Im Folgenden wird gefragt, für welche Texte und Teiltexte dieses Verwendungsprinzip typisch und konstitutiv ist.

Einer ersten Stufe des Verfahrens begegnet man des öfteren in Zeitungsüberschriften, vorzugsweise in der Rubrik „Vermischte Meldungen“ oder auf der Lokalseite. Dieses Verfahren ist häufig beschrieben worden; es sei daher nur mit einem einzigen Beispiel in Erinnerung gerufen, um auf die Darstellung einer intensiveren Nutzung des Verfahrens im Sinne eines textkonstitutiven Prinzips vorzubereiten:

(16) Türken kommen auf den Hund (Hildesheimer Allgemeine Zeitung, Lokalteil, 3.1.03, S. 13)

Textinhalt: Türkische Jugendliche helfen bei der Suche nach einem verloren gegangenen Hund und finden ihn.

19 Berichtenswert erschien der taz beispielsweise folgender Fall von „Ethnomarketing“, der in dem beschriebenen Sinne „unfreiwillig komisch“ ist: „Kaum ein deutsches Unternehmen kümmert sich bislang um die Deutschtürken als Konsumenten. [...] Und so manche Kampagne scheiterte am Unwissen über kulturelle Eigenheiten. So warb der Telefonanbieter TelDaFax mit dem Slogan Kein Schwein ruft mich an und erntete bei den muslimischen Türken damit freilich keine Pluspunkte" (taz 10.7.2002, S. 9). TelDaFax hat sich offenbar nicht klar gemacht, dass die Bildlichkeit von Idiomen aktualisierbar und die Verwendung dieses spezifischen Phrasems für die Adressaten daher offenkundig ungeeignet ist. Der berichtende „Sprecher“, die taz, „weiß es besser“" und entlarvt den Originalsprecher in seiner Naivität und Unwissenheit. 
Die Überschrift nutzt nur den Wortlaut des Phrasems auf den Hund kommen und formuliert damit das Thema des Textes. Es handelt sich freilich auch hier nicht um ein einfaches Wörtlichnehmen, denn der Wortlaut muss in textbezogener Weise umgedeutet werden: auf etwas kommen ist hier auf das Finden von etwas Konkretem zu beziehen, während die Verbkonstruktion üblicherweise im gedanklichen Sinne verwendet wird (,jdm einfallen'). ${ }^{20}$

Entscheidend ist: die Phrasembedeutung ist für den Inhalt des Textes nicht relevant. Aus der Sicht des Phrasems als Einheit gibt es lediglich ausdrucksseitige Beziehungen zwischen Phrasem und Text, d.h. es wird die komplexe signifiant-Seite der Mehrwortverbindung genutzt. So gesehen gibt es (lediglich) eine „kohäsive Brücke“ zwischen Überschrift und Text. ${ }^{21}$

Dasselbe Verfahren nun kann intensiver genutzt werden. Das bedeutet, dass sich weder die Phrasembedeutung noch die - oder eine - wörtliche Lesart in eine kohärente Beziehung zur Textbedeutung bringen lassen; vielmehr erfolgt die Bindung nur noch lockerer über eine andere wörtliche Bedeutung einer einzelnen Komponente. Solche linguistisch lockeren - weil vornehmlich kohäsiven, nur über die Ausdrucksseite erstellten - Verknüpfungen begegnen zum einen als punktuelle, lokal begrenzte „Auflockerung“ des Textes, die diesem eine bestimmte Qualität verleiht. Zum anderen werden sie, in einer gesteigerten Form, zum konstitutiven Prinzip eines ,leicht und locker gestrickten Textes“. In beiden Fällen weichen die Texte bewusst vom guten Pfad strenger Rationalität ab.

Mit (17) liegt ein Beispiel für eine punktuelle Auflockerung des Textes vor:

(17) Bericht von der Grünen Woche in Berlin; die Teiltexte sind alphabetisch geordnet.

K wie Kängurufleisch: Probieren Sie ruhig mal!

Während der BSE-Krise ist das Fleisch der symbolträchtigen Tiere nix im Beutel, aber große Sprünge machen ${ }^{22}$ - zusammen mit Kroko-

${ }^{20}$ Hier zeigt sich ein weiteres Mal, dass die häufig verwendete und aus der ein-Wortzentrierten Linguistik übernommene Ausdrucksweise „Ambiguierung (eines Phrasems)" zur Benennung dieses Verfahrens streng genommen nicht richtig ist; sie hat sich aber weitgehend eingebürgert; jede andere Art der Bezeichnung wäre umständlich und wenig praktikabel.

${ }^{21}$ Auch die Begriffe Kohäsion und Isotopie wurzein in der ein-Wort-zentrierten Linguistik (siehe auch die letzte Anmerkung). Sie beziehen sich typischerweise auf Lexeme und eine einzige Bedeutungsebene. Das Verständnis der Begriffe müsste erweitert werden, um komplexe phraseologische Zeichen mit einer doppelten Ebene der Signifikation mit einzubeziehen. Isotopieketten und kohäsive Bezüge in einem so erweiterten Sinne entstehen durch rekurrente Wörter, die auf unterschiedlichen Bedeutungsebenen zu lesen sind und zeichentheoretisch einen unterschiedlichen Status haben.

${ }^{22} \mathrm{Vgl}$. im einzelnen die Wendungen tief in den Beutel greifen müssen (ugs.), ,viel zahlen müssen'; auch: ein großes/arges Loch in jmds. Beutel reißen; den Beutel ziehen; der Beutel ist leer $($ Beutel $=$,Geldbeutel'); keine großen Sprünge machen können 
dilschnitzeln und Straußensteaks in Mode gekommen. (taz 21.1.2003, S. 5)

Die eingeschobene Bemerkung unterbricht den Text tatsächlich und lockert ihn in einem ganz konkreten Sinne auf. Zitiert wird ein Teil eines phraseologischen Vergleichs für Armut, der hauptsächlich in der gesprochenen Umgangssprache im Umlauf ist: wie ein Känguru - nix im Beutel, aber große Sprünge machen..$^{23}$ Dieser Vergleich ist auf Grund des kaum entwirrbaren Wechsels zwischen den Bedeutungsebenen, zwischen tierischer und menschlicher Welt, und auf Grund der Kombination von Wörtlichkeit, surreal-phantastischer Motivierung und Vermenschlichung schon in sich komisch (nix im (Känguru-)Beutel - nichts im (Geld-)beutel; große Sprünge des Kängurus - große finanzielle Sprünge). Anders als in (16) ist hier jedoch keine der beiden Bedeutungsebenen für die Textbedeutung relevant. Weder ist es im Textzusammenhang sinnvoll, an Aussehen und Fähigkeiten des Kängurus zu erinnern, noch gibt es einen Sinn, finanzielle Verhältnisse von Kängurus zu thematisieren. Der Einschub ist folglich nur durch bloße Kohäsion wörtlich zu lesender Ausdrücke lokal an den Text angebunden. Der Einschub bezieht allein daraus seine Legitimation, seine Funktion liegt auf einer anderen Ebene.

Zum konstituierenden Prinzip, die eine lokal begrenzte „Auflockerung“ eines Textes überschreitet, wird das Verfahren in moderierenden Texten in Radio und Fernsehen, welcher sich "locker" geben wollen. Moderationen haben in jüngerer Zeit an Bedeutung gewonnen. Dank ihrer klaren Grenzen und bestimmbaren Funktion, einen Sendebeitrag oder Gesprächspartner anzukündigen, können sie als eigenständige Texte bezeichnet werden. ${ }^{24}$ Dass Moderationen hier im Zusammenhang mit schriftlichen Texten erwähnt werden, lässt sich im Sinne der Unterscheidung von Konzeption (hier: schriftlich) und Medium (hier: gesprochen; siehe Koch/Oesterreicher 1990, S. $5 \mathrm{ff}$.) dadurch rechtfertigen, dass Moderationen oftmals im Voraus konzipiert und dabei oft auch teilweise aufgeschrieben werden.

Auch diese Texte sind nur locker gewebt. Die Sätze und Teilsätze werden oftmals überhaupt nur dank des Einsatzes eines Phrasems oberflächlich zusammengehalten. Mit Kriterien inhaltlicher Stimmigkeit und logischer Folgerichtigkeit, die in der Linguistik bei der Beschreibung von Texten generell zugrunde gelegt werden, ist diesen Texten nicht beizukommen. Ein kurzes Beispiel:

(umgs.), typisch in negierter Form: ,keine großen finanziellen Mittel zur Verfügung haben' (Duden Band 11).

${ }^{23}$ Drei Belege zu diesem Ausdruck mit leichten Ausdrucksvarianten finden sich im Korpus von Elisabeth Piirainen zu Phraseologismen in der deutschen Umgangssprache, auf der ihre Publikation aus dem Jahr 2002 basiert. Ich bedanke mich an dieser Stelle herzlich für die entsprechende Mitteilung.

${ }^{24} \mathrm{Zu}$ einer Analyse magazinartiger Fernsehnachrichten auf dem Hintergrund des Trends zum sogenannten Infotainment siehe Burger (1999). 
(18) Sprecherin zwischen zwei Musikstücken:

Die Zeit jetzt: 20 Minuten nach 3, gleich kommt wieder unser Musikrätsel, und obwohl wir ja seit gestern nach Auffassung der Meteorologen Frühling haben, diesmal könnte Sie die Rückwärtsmusik aufs Glatteis führen. In knapp 10 Minuten wissen wir mehr. [Ende; es folgt Musik] (28.2.2003, NDR Kultur)

Erläuterung zu ,Rückwärtsmusik‘: Die Rätselaufgabe besteht im Erraten eines rückwärts gespielten Musikstücks.

Der Inhalt der Moderation besteht weitgehend in einer Motivierung des Phrasems aufs Glatteis führen. $\mathrm{Zu}$ diesem Zweck wird der angebrochene Frühling erwähnt, also ein Element der Textsituation, und dort wiederum das unausweichliche und allgegenwärtige, insofern auch banale Wetter. Frühling schafft als Teil des Frames Jahreszeiten eine Verbindung zum Winter und zum (wörtlichen) Glatteis in der Wendung aufs Glatteis führen. Ein logischer Zusammenhang zwischen Frühling und aufs Glatteis führen wird somit sprachlich suggeriert, ist aber faktisch nicht gegeben. Selbst eine thematische Überleitung zum Musikstück leistet der Text nicht, da die nachfolgende Musik weder etwas mit dem Frühling noch mit dem Winter (oder einer anderen Jahreszeit) zu tun hat. Die lockere Zusammenführung von Frühling und aufs Glatteis führen scheint somit die einzige Motivation für den Text, der sich einer weiteren rationalen Deutung entzieht. Solche nichts-sagenden Texte gelten offenbar als „leicht und locker“. Es gibt auch längere Moderationen, die nach demselben Muster gestrickt sind. ${ }^{25}$

\section{Gehäufte Verwendungen als textkonstituierendes Prinzip}

Häufungen bestimmter Ausdrücke oder Verwendungsweisen können konstitutiv für bestimmte Textsorten und deren Funktionen sein oder zumindest einen entscheidenden Anteil daran haben. ${ }^{26}$ Auch hier ist grundsätzlich zwischen dem Verfahren und der damit verbundenen Aussageabsicht zu unterscheiden, die sich in bestimmten Textsorten bzw. deren Funktionen manifestiert. Wenngleich keine eindeutigen Korrelationen in einem streng statistischen Sinne „berechenbar" sind, so lassen sich doch auch hier (vgl. oben, Abschnitt 2.1) einige Tendenzen ausmachen: bestimmten Verfahren kann ein typisches Profil von Funktionen zugeordnet werden.

${ }^{25}$ Derartige Moderationen tauchen seit Einführung eines neuen Sendeformats bei NDR Kultur zum 1.1.2003, das explizit einen höheren Unterhaltungswert im Vergleich zum früheren Format anstrebt, verstärkt auf. Das Programm hat sich seither bezeichnenderweise auch den doppelbödigen Beinamen Der Klassiker gegeben.

${ }^{26}$ In dem ursprünglichen Konzept der ,textbildenden Potenzen“" entsprechen Häufungen der "höchsten Stufe" dieser Potenzen. Allerdings wurde dort nur das Prinzip an sich beschrieben, also die Möglichkeit, dass der Einsatz von Phrasemen und auffälligen Verwendungen zum ,textorganisierenden Prinzip schlechthin“ werden kann. Eine Verbindung zu typischen Textfunktionen wurde nicht hergestellt. 
Diese Verfahren können auf Grund der Unterscheidungen, die eingangs getroffen wurden und die, im Einklang mit dem ursprünglichen Konzept der textbildenen Potenzen, in der Beschaffenheit der Phraseme begründet sind, präzisiert werden, $d . h$. es kann angegeben werden, was genau wiederholt und gehäuft verwendet wird. Demnach lassen sich drei Möglichkeiten unterscheiden:

1. Eine Häufung bestimmter Verwendungsweisen, der „kohäsiven Brücken "; diese ist, wie in 5.3 beschrieben, ein Kennzeichen eines „lockeren" Textes, wie er in bestimmten Moderationen in Radio und Fernsehen anzutreffen ist.

2. Eine Häufung eines bestimmten Phrasemtyps; diese kann eine Distanz zu genau diesem Ausdrucksmittel ausdrücken und somit einen wesentlichen Anteil an satirisch-ironisierenden Redeweisen haben. Ein Beispiel ist der Sketch Die Bundestagsrede von Loriot. Hier ein Auszug:

(19) Moderator: [...] Der zur Zeit parteilose Abgeordnete Werner Bornheim hielt eine Rede, die für einen neuen politischen Stil richtungsweisend sein könnte. [...]

W. Bornheim: Meine Damen und Herren, Politik bedeutet, und davon sollte man ausgehen, das ist doch - ohne darumherumzureden - in Anbetracht der Situation, in der wir uns befinden. Ich kann meinen politischen Standpunkt in wenige Worte zusammenfassen: Erstens das Selbstverständnis unter der Voraussetzung, zweitens, und das ist es, was wir unseren Wählern schuldig sind, drittens, die konzentrierte Be-inhaltung als Kernstück eines zukunftsweisenden Parteiprogramms.

Wer hat denn, und das muss vor diesem hohen Hause einmal unmißverständlich ausgesprochen werden. [...] (Loriots dramatische Werke, Zürich 1990)

Fast der gesamte Text ist, einer Collage gleich, aus schlagwortartigen Versatzstücken gebaut. Darunter befinden sich zahlreiche textstrukturierende Formeln und rhetorische Formeln öffentlicher Reden, auch in nur angedeuteter oder verzerrter, syntaktisch unvollständiger Form. Die Überzeichnung durch übermäßige Nutzung der Formeln lässt diese als Leerformeln erscheinen und gibt sie der Lächerlichkeit preis.

3. Eine Häufung von Phrasemen mit einem bestimmten thematischen Kern.

Der folgende Text, eine ironische Glosse zur Flutkatastrophe im Jahr 2002, enthält verschiedene feste Wendungen und Anspielungen mit einem gleichen oder ähnlichen Bildkern, arbeitet darüber hinaus aber auch mit passenden einfachen Wörtern und Komposita aus demselben Wort- und Bildfeld. Insgesamt kommt es zu einer Verdichtung der Isotopien im erweiterten Sinne, welche Wortfeld und Bildfeld betrifft und sich auch nur auf einzelne Bestandteile (Wortbildungsbestandteile; Phrasemkomponenten) richten kann. 
Außerdem enthält der Text noch weitere Sprachmittel, um seine distanziertspöttische Qualität zu erreichen.

(20) Die deutsche Woge

Die verheerenden Überschwemmungen der vergangenen Wochen haben auch einen nicht einzudämmenden Wortschwall über uns hereinbrechen lassen. Wenn denn irgendwann die Gesammelten Reden unserer derzeitigen Wahlkämpfer erscheinen, dann werden die Beiträge aus dem August 2002 mutmaßlich jeweils eigene Bände mit Goldschnitt füllen, denn es waren goldene Worte, die wir hören durften, und die Goldgräberstimmung der Redenschreiber hielt an bis gestern, als der Bundestag sich nach gebührender Wartezeit - alle seine in den überfluteten $\mathrm{Ge}-$ bieten aktiven Mitglieder sollten brav ausreden dürfen - versammelte, um die angekündigte Soforthilfe abzusegnen, auf daß es keinem Opfer materiell schlechter als vor der Katastrophe gehen möge. Ideell geht es uns allen schon viel besser. Denn wir haben uns erwiesen als ein einig Volk von Brüdern, in keiner Not uns getrennt und Gefahr. Das haben wir nun mündlich, gesprochen vom Kanzler, geerntet auf dem überreifen Metaphernfeld, das mit einem Mal so gut bestellt ward: „Durch Deutschland ist nicht nur eine katastrophale Flut gegangen, sondern auch eine unglaubliche Welle der Solidarität." Vergessen ist der deutsche Weg, es lebe lang die deutsche Woge. [...] Da steht der Überschwemmte nun in Sachsen, Bayern, Brandenburg, vor sich die Nachbarn und hinter sich die ganze Welt - und doch fehlt etwas über ihm. [...] (FAZ, Feuilleton, 30.8.2002, S. 33)

Dieses letzte Textbeispiel zeigt erneut (siehe oben, Abschnitt 4.), dass Phraseme und Phrasemverwendungen zwar ein wichtiges Ausdrucksmittel sind, dass es aber bei textbezogenen funktionalen Fragestellungen oftmals sinnvoll ist, auch andere Sprachmittel miteinzubeziehen. ${ }^{27}$

\section{Schlussfolgerungen und Ausblick}

Die Rolle von Phrasemen für die „Konstitution“ und „Funktion“ von Texten, wie sie - ausschnittweise - beschrieben wurden, legt nahe, über einige grundsätzliche Vorstellungen vom Text in der Linguistik nachzudenken:

1. Wie gezeigt wurde, sind bestimmte Phrasemverwendungen oft konstitutiv für Texte mit bestimmten affektiven Dimensionen. Zu solchen Texten gehören die Witzzeichnung ebenso wie der ironisierende Sketch und die distanziert-spöttische Glosse oder auch die journalistische Karikatur als bildlich-textliche Form des Kommentars. Die mit diesen Texten verbundenen affektiven Dimensionen können vielfältig sein: der Leser kann belustigt sein

27 Entsprechende Untersuchungen können auch für literarische Texte aufschlussreich sein, wie sie etwa mit Fernandez Bravo (2001) vorliegen. 
und sich ,unterhalten“ fühlen, er kann sich beim Erkennen einer Anspielung „bewandert" und dadurch ,aufgewertet" fühlen, er kann in den ironisierenden Spott des Verfassers, der ,aus dessen Text spricht", innerlich ,mit einstimmen" oder zu einer Gegenreaktion herausgefordert werden. Auf der Seite des Textautors kann man ein gewisses „Vergnügen“ vermuten, das er (oder sie) an einer Formulierung, am Texteschreiben gefunden hat.

Für dieses Funktionsspektrum nun steht in der Linguistik kein rechter Terminus zur Verfügung. Das liegt möglicherweise daran, dass Auffassungen vom Text in der Linguistik nach wie vor „sehr stark durch den als Prototyp gesetzten Fall der zweckrationalen Kommunikation geprägt sind“ (Adamzik 2001, S. 280). ${ }^{28}$

2. Mit dem eben Genannten hängt ein Zweites zusammen: In unserer Vorstellung von einem prototypischen Text spielt die Kohärenz, der inhaltlichthematische Zusammenhang und die semantische Konstanz der Ausdrucksmittel, eine zentrale Rolle. Auch Sprachteilhaber selbst orientieren sich bei ihrer Bewertung eines Sprachausschnitts ,als Text" an diesem Kriterium (Adamzik 2001, S. 258). Dem sind die empirisch vorfindbaren Zeichenfolgen vom Typ der "lockeren Texte" entgegenzuhalten (Abschnitt 5.3.), bei denen die Kohärenz eine geringere Rolle spielt, die aber offenbar von den Sprachteilhabern als Texte aufgefasst werden. Es ergibt sich die Frage, wie diese empirische Wirklichkeit theoretisch berücksichtigt werden kann.

3. Die Witzzeichnungen schließlich legen ein Weiteres nahe: Hier ist es in manchen Fällen wahrscheinlich weniger so, dass für einen Gedanken, eine Idee nach einem zeichnerischen Ausdrucksmittel gesucht wurde, als vielmehr umgekehrt: die Sprache, ein Phrasem, ist das auslösende Moment, gibt die Inspiration für den Entwurf einer - hier zeichnerischen - Situation (letztere ist genausogut als Geschichte denkbar). Dies sollte zum Einen Anlass sein, das Intermediale - die Bild-Text-Relation, die in idiomatischen Phrasemen ja selbst angelegt ist - von Seiten der Linguistik stärker zu berücksichtigen. Zum Zweiten aber weisen solche Texte auf die kognitive Kraft von Phrasemen für den Prozess des Text-Erstellens. Das Konzept der textbildenden Potenzen, das in diesem Beitrag weiter gefüllt wurde und sich dominant am Text als Produkt orientiert, könnte durch dieses kognitive und prozessbezogene Moment ergänzt werden. Dann ließe sich möglicherweise auch eine deutlichere Brücke zu literarischen Verarbeitungen von Phrasemen, beispielsweise in Aphorismen oder Sprichwortgedichten, schlagen.

${ }^{28}$ Weitere Beispiele in der Linguistik für die Dominanz des rationalen und Nützlichkeitsgedankens (im Sinne des lat. prodesse im Unterschied zum delectare) sind: die Übertragung des Rasters illokutiver Funktionen auf Textfunktionen, in der kein Platz für affektive Dimensionen ist (etwa bei Brinker 2001, Kap 4.), oder auch Vorstellungen vom Textherstellen als einer „Problemlösungsstrategie“. 


\section{Literatur}

Adamzik, Kirsten (2001): Sprache: Wege zum Verstehen. Tübingen/Basel.

Balsliemke, Petra (2001): ,Da sieht die Welt schon anders aus.'Phraseologismen in der Anzeigenwerbung. Modifikation und Funktion in Text-Bild-Beziehungen. Baltmannsweiler. (Phraseologie und Parömiologie 7.)

Blumenthal, Peter (1999): Schématismes dans les commentaires de presse allemands et français. In: Nouveaux Cahiers d'allemand 17, S. 577-593.

Brinker, Klaus (2001): Linguistische Textanalyse. 5. durchges. u. erg. Aufl. Berlin.

Burger, Harald (1989): ,Bildhaft, übertragen, metaphorisch ... ' Zur Konfusion um die semantischen Merkmale von Phraseologismen. In: Gréciano, Gertrud (Hg.): Europhras 1988. Phraséologie Contrastive. Strasbourg. S. 17-29.

Burger, Harald (1998): Phraseologie: Eine Einführung am Beispiel des Deutschen. Berlin. Burger, Harald (1999): Phraseologie in Fernsehnachrichten. In: Baur, Rupprecht S. et al. (Hg.): Wörter in Bildern - Bilder in Wörtern. Beiträge zur Phraseologie und Sprichwortforschung aus dem Westfälischen Arbeitskreis. Baltmannsweiler. (Phraseologie und Parömiologie 1.) S. 71-106.

Cortès, Colette (2001): ,Sozusagen im doppelten Sinne'. Zur Klassifizierung metasprachlich autonymisch verwendeter Phraseme im Deutschen. In: Häcki Buhofer, Annelies u. a. (Hg.): Phraseologiae amor. Aspekte europäischer Phraseologie. Baltmannsweiler. (Phraseologie und Parömiologie 8.) S. 43-61.

Cowie, Anthony P. (1991): Multiword Units in Newspaper Language. In: Granger, Sylviane $(\mathrm{Hg}$.): Perspectives on the English lexicon: a tribute to Jacques van Roey. Cahiers de l'Institut de Linguistique de Louvain. Louvain-la-Neuve. S. 101-116.

Dobrovol'skij, Dmitrij O. (1980): Zur Dialektik des Begriffs der textbildenden Potenzen von Phraseologismen. In: Zeitschrift für Phonetik, Sprachwissenschaft und Kommunikationsforschung 33.6, S. 690-700.

Dobrovol'skij, Dmitrij O. (1987): Textbildende Potenzen von Phraseologismen. In: Linguistische Studien ZISW/A 164, S. 69-85.

Donalies, Elke (1994): Idiom, Phraseologismus oder Phrasem? Zum Oberbegriff eines Bereichs der Linguistik. In: Zeitschrift für germanistische Linguistik 22, S. 334-349.

Duden Band 11 = Duden Redewendungen. Wörterbuch der deutschen Idiomatik (2002): 2., neu bearb. und aktualisierte Aufl. Mannheim u. a.

Fernandez Bravo, Nicole (2001): Rhetorik und Phraseologie im Text,Das Lied von der großen Kapitulation' (in Bertolt Brechts ,Mutter Courage und ihre Kinder'). In: Häcki Buhofer, Annelies u. a. (Hg.): Phraseologiae amor. Aspekte europäischer Phraseologie. Baltmannsweiler. (Phraseologie und Parömiologie 8.) S. 135-149.

Das Fischer Lexikon Publizistik, Massenkommunikation (1995), hg. Elisabeth NoelleNeumann u. a. Aktual., vollst. überarb. Neuausgabe. Frankfurt a. M.

Fleischer, Wolfgang (1997): Phraseologie der deutschen Gegenwartsprache. 2., durchges. u. erg. Aufl. Tübingen.

Granger, Sylviane (1998): Prefabricated Patterns in Advanced EFL Writing: Collocations and Formulae. In: Cowie, Anthony P. (Hg.): Phraseology: Theory, Analysis, and Applications. Oxford. S. 145-160.

Hemmi, Andrea (1994): ,Es muss wirksam werben, wer nicht will verderben'. Kontrastive Analyse von Phraseologismen in Anzeigen-, Radio- und Fernsehwerbung. Bern.

Koch, Peter/Oesterreicher, Wulf (1990): Gesprochene Sprache in der Romania: Französisch, Italienisch, Spanisch. Tübingen.

Landheer, Ronald (1989): L'ambiguïté: un défi traductologique. In: Meta 34.1, S. 33-43. Piirainen, Elisabeth (2002): Er zahlt keine Steuern mehr. Phraseologismen für ,sterben` 
in den deutschen Umgangssprachen. In: Piirainen, Elisabeth/Piirainen, Ilpo Tapani (Hg.): Phraseologie in Raum und Zeit. Akten der 10. Tagung des Westfälischen Arbeitskreises „Phraseologie/Parömiologie“ (Münster 2001). Baltmannsweiler. (Phraseologie und Parömiologie 10.) S. 213-238.

Sabban, Annette (1998a): Okkasionelle Variationen sprachlicher Schematismen. Eine Analyse französischer und deutscher Presse- und Werbetexte. Tübingen.

Sabban, Annette (1998b): Negative orientation in advertising as a pragmatic paradox - an aspect of the contemporary use of idioms. In: Durčo, Peter (Hg.): Europhras 97. Bratislava. S. 313-320.

Sabban, Annette (1998c): ,Fühlen Sie sich nur nicht angesprochen!‘ Inszenierte Negativität in der Werbung. In: Wirrer, Jan (Hg.): Phraseologismen in Text und Kontext. Bielefeld. (Phrasemata I.) S. 73-95.

Sabban, Annette (Hg.) (1999): Phraseologie und Übersetzen. Bielefeld. (Phrasemata II.) Sabban, Annette (2001): A propos de l'ambiguïsation dans le discours. In: Bogaards, Paul/ Rooryck, Johan/Smith, Paul J. (Hg.), avec la collaboration de Véronique van Gelderen. Quitte ou double sens: articles sur l'ambiguité offerts à Ronald Landheer. Amsterdam u. a. S. 305-320.

Schemann, Hans (1993): Deutsche Idiomatik: Die deutschen Redewendungen im Kontext. Stuttgart u. a.

Sinclair, John (1991): Corpus, concordance, collocation. Oxford u. a.

Sowinski, Bernhard (1998): Werbung. Tübingen. (Grundlagen der Medienkommunikation 4.)

Steyer, Kathrin (2003): Korpus, Statistik, Kookkurrenz. Lässt sich Idiomatisches ,berechnen'? In: Burger, Harald u.a. (Hg.): Flut von Texten - Vielfalt der Kulturen. Baltmannsweiler. (Phraseologie und Parömiologie 14.) S. 33-46.

Wotjak, Barbara (1992): Verbale Phraseolexeme in System und Text. Tübingen. 